\title{
Eveil de la conscience grammaticale chez un enfant français entre 18 mois et 3 ans
}

\author{
Marie Leroy-Collombel \\ Modyco - Université Paris Descartes, CNRS, Université Paris Ouest Nanterre La Défense \\ marie.leroy@parisdescartes.fr
}

\section{Introduction}

Quand et comment le petit enfant entre-t-il dans la grammaire de sa langue ? Acquérir la grammaire, c'est apprendre à maîtriser l'usage des marqueurs morphologiques de sa langue maternelle (Karmiloff \& Karmiloff-Smith, 2001), à combiner les unités à l'intérieur de la phrase, et à combiner les phrases entre elles. Pour apprendre la grammaire, l'enfant doit donc être attentif à plusieurs niveaux de langue en même temps : le niveau sémantique (il doit apprendre à relier des "mots» à des référents), le niveau morphologique et le niveau syntaxique (agencement des mots et des propositions dans les phrases).

Les langues fournissent deux sortes d'éléments signifiants, et utilisent les deux pour créer des constructions grammaticales (Slobin, 2001) : des morphèmes qui font référence aux objets et événements de l'expérience, et des morphèmes qui relient ces morceaux d'expérience les uns aux autres et aux perspectives de discours du locuteur. On oppose ainsi le contenu à la relation, les items lexicaux aux items grammaticaux, les mots à contenu aux mots fonctionnels. Parmi les premiers, on trouvera les noms, les verbes, les adjectifs et les adverbes, et dans la seconde catégorie, les morphèmes libres ou non liés (conjonctions, prépositions) et les morphèmes liés (affixes marquant le nombre, le cas, le temps...).

Par conséquent, l'enfant doit acquérir différents types de morphèmes, tant au niveau de leur signifiant que de leur signifié, et apprendre à les combiner pour former des unités de la langue.

Quand et comment les enfants commencent-ils à utiliser les différents types de morphèmes et que peut-on en déduire concernant les processus d'acquisition du langage?

\subsection{Etapes dans la mise en place de la morphologie}

La perception de la morphologie semble en précéder la production. Ainsi, Gerken (1987) a démontré par des expériences de répétition de phrases avec des enfant de 26 mois, qu'à un stade où les enfants ne produisent pas encore de termes fonctionnels, ils sont néanmoins sensibles à leur présence et à leur distribution. Lorsque les enfants avec des MLU (Mean Lenght of Utterance ou longueur moyenne d'énoncés, $c f$. Mc Whinney, 2000) peu élevés répètent des phrases, ils omettent les fonctionnels qui entourent les mots lexicaux familiers. Les enfants considèreraient les fonctionnels, notamment à cause de leur manque de saillance phonétique, comme des éléments familiers mais faiblement spécifiés, constituant des sortes de «cadres» ( frames ») fournissant des « emplacements » (《slots 》) pour des mots lexicaux, plus saillants au niveau phonologique et sémantique.

On retrouve cette idée chez Dressler (1995) qui propose plusieurs étapes dans l'acquisition de la morphologie :

- un stade prémorphologique au cours duquel les enfants produisent des énoncés contenant plus d'une unité, mais sans que les combinaisons ne soient systématiques. Il s'agirait d'opérations locales, comme des réduplications d'unités ou l'adjonction de fillers (cf. notamment Peters 2001 et Veneziano \& Sinclair 2000 sur ce point), que l'enfant n'étendrait pas de manière plus large dans un premier temps ; 
- Un stade protomorphologique, au cours duquel l'enfant commence à développer un système de grammaire morphologique. Il analyse des unités qui étaient auparavant non analysées, il étend ses analogies à plus d'une forme, mais reste limité dans sa productivité. Il met en place des " proto-règles », spécifiques à certaines formes et devient sensible aux positions à l'intérieur des énoncés ;

- Et enfin, un stade morphologique à part entière ( full morphology»), avec des règles combinatoires systématiques.

\subsection{Construire la grammaire de sa langue?}

On peut expliquer la façon dont l'enfant accède à ce stade morphologique de plusieurs manières, reflétées dans les différents courants théoriques. Soit nous considérons, comme Chomsky, que l'enfant est doté à la naissance de connaissances structurelles (la «grammaire universelle») lui permettant de produire les énoncés conformes à la grammaire de sa langue maternelle à partir d'une exposition minimale, mais suffisante, pour régler certains paramètres spécifiques à cette langue, soit nous considérons que l'enfant construit par lui-même un système de relation entre forme et fonction, à partir de son observation de l'input langagier. Pour Tomasello (2003), par exemple, la compétence linguistique ne correspond pas à la maitrise d'une grammaire formelle reposant sur des règles sémantiquement vides, mais plutôt à la maitrise d'un inventaire structurel de constructions linguistiquement signifiantes. Dans un premier temps, ces constructions sont des îlots relativement isolés, et se fondent sur des unités particulières. Le processus d'acquisition de la grammaire relèverait ainsi d'une construction progressive de règles, à partir de l'usage (" usage-based theories ») : les enfants construiraient des règles abstraites à partir de leur compréhension des fonctions communicatives incarnées par diverses constructions syntaxiques, en déchiffrant les intentions du locuteur, à partir de capacités cognitives générales. A partir de constructions isolées («itembased constructions »), les enfants déduiraient des modèles, des règles, par schématisation et analogie.

Pour Tomasello (2003), les enfants procèdent en deux étapes pour acquérir la grammaire de leur langue :

- à partir des énoncés et expressions, ils extraient de petits «éléments» - des mots, des morphèmes, des phrases - en identifiant leur rôle dans la communication ("communicative $j o b »)$

- ils repèrent des patterns, des modèles, à travers les énoncés (ou parties d'énoncés), avec des structures et des fonctions similaires, qui leur permettent de créer à leur tour des catégories et des constructions plus ou moins abstraites.

Les enfants utiliseraient ces modèles constructionnels comme des gabarits («templates ») à l'intérieur desquels ils inséreraient les mots, morphèmes et phrases extraits précédemment, pour créer leurs propres énoncés. L'enfant s'appuierait donc sur le sens des énoncés de l'adulte, à partir des intentions de communication de ce dernier. L'acquisition des mots et l'acquisition des constructions grammaticales relèveraient d'un même processus, dans la mesure où ils sont les uns comme les autres des symboles linguistiques porteurs de sens, même s'ils se situent à des niveaux de complexité différents. L'acquisition de la grammaire serait par conséquent progressive, irrégulière et dépendante du lexique. L'importance du lexique est également soulignée par Bassano (2003), pour laquelle le développement grammatical précoce dépend du développement lexical, et plus spécifiquement d'une «masse lexicale critique » nécessaire pour que la grammaticalisation puisse s'effectuer.

On peut donc supposer que l'enfant construit son propre système de règles, à partir de l'input qu'il reçoit de son entourage, et qu'il rectifie ses règles au fur et à mesure, aidé en cela par certains procédés comme la reformulation par l'adulte de ses productions «incorrectes», non conformes à la grammaire, reformulations qui constituent des corrections implicites, intégrées dans l'énoncé de l'adulte (cf. Chouinard \& Clark, 2000). Cette capacité à construire des règles grammaticales implicites ferait défaut aux enfants dysphasiques (Gopnik, 1990). 
Pour Bowerman (1985), les premiers usages appropriés d'une forme par l'enfant sont souvent fondés sur une connaissance superficielle. En effet, les premières productions ne sont pas encore analysées. Ce n'est que dans un second temps que l'enfant découvre des règles et essaie de les appliquer, en les surgénéralisant, avant de revenir à une utilisation correcte une fois qu'il a intégré les règles et leurs exceptions (hypothèse de la "courbe en U », cf. Karmiloff \& Karmiloff-Smith, 2001). L'acquisition serait par conséquent un processus laborieux, de longue haleine, au cours duquel l'enfant découvrirait successivement des niveaux de structure et de régularité plus profonds, au niveau de la forme et du sens. En d'autres termes, l'enfant affinerait progressivement, au fur et à mesure de son développement, son analyse du système de sa langue, et par conséquent son système de règles. Le temps d'acquisition d'une structure dépendrait de plusieurs facteurs : sa complexité cognitive et linguistique, son utilité pragmatique et sa fréquence (Bowerman, 1985).

Comme nous l'avons mentionné plus haut, Tomasello (2003) suggère l'existence de deux faces ou aspects dans la grammaire : d'un côté, de petits éléments, et de l'autre, de larges "patterns », l'objectif étant de combiner les petits éléments à l'intérieur des patterns. Le premier problème pour l'enfant va donc être d'isoler les parties ou éléments sur lesquels «travailler », de repérer les contextes dans lesquels ces éléments sont appropriés, et d'apprendre à construire de nouveaux blocs à partir d'éléments anciens (Nelson, 1981). L'enfant ne va pas construire son langage en analysant séparément les niveaux linguistiques (lexique, syntaxe, phonologie et pragmatique), mais va acquérir des petits morceaux de chacun de ces niveaux de manière simultanée (Nelson, 1981).

\subsection{Des trajectoires différentes}

Afin de parvenir à l'objectif de combinaison de petits éléments de sens (les morphèmes) à l'intérieur de «blocs», à la manière d'un puzzle se construisant progressivement, les enfants pourront suivre des trajectoires différentes, utiliser des stratégies individuelles, de la même manière qu'un puzzle qu'on peut commencer par les bords, ou par le milieu, ou dont on peut trier les pièces par couleurs ou par formes (Leroy \& Martel, sous presse). Les différences individuelles sont importantes dans le processus d'acquisition et dans le langage produit par les enfants entre un et cinq ans, ce qui pourrait s'expliquer, selon K. Nelson (1981), par la construction de différents systèmes de règles. Les différentes stratégies correspondent à des portes d'entrée différentes dans la grammaire. Selon les auteurs, les terminologies diffèrent, mais l'idée reste la même : certains enfants commencent par les petits éléments, alors que d'autres vont privilégier les «blocs». On opposera ainsi les enfants produisant des mots à ceux produisant des phrases, parlant de styles référentiel versus expressif, cognitif versus pragmatique, ou encore analytique versus gestalt. La stratégie «globale» consiste à entrer dans le langage à partir des « patterns » larges, des blocs, non analysés. Ces blocs sont appelés « gestalt language » (Peters, 1977), « stereotype units» (Nelson, 1981) ou encore « figements » (François, 1977). Selon Nelson (1981), ces différences individuelles peuvent s'expliquer au moyen de la théorie des trois facteurs du développement symbolique («3-factor theory») : deux facteurs cognitifs entrent en jeu, l'analytique et le global, auxquels s'ajoute l'intention de communication, et c'est seulement lorsque ces trois composantes sont intégrées que le langage émerge. Les différences individuelles pourraient résulter d'un développement asymétrique des différentes composantes (qui seraient gérées par l'hémisphère gauche du cerveau pour la partie analytique, et l'hémisphère droit pour la partie globale). Les différences peuvent également être déterminées par les conditions environnementales d'apprentissage : le niveau d'éducation, l'ordre dans la fratrie, la catégorie sociale, le type de langage adressé à l'enfant, sont autant de facteurs qui peuvent influencer la façon dont l'enfant acquiert le langage. Nelson ajoute que les enfants adoptent des stratégies et des styles différents parce qu'ils ont des hypothèses différentes sur ce à quoi sert le langage.

L'enfant est confronté à un matériau complexe, le langage, qu'il lui faut s'approprier, sur tous les fronts en même temps : phonologique, syntaxique, morphologique, lexical et pragmatique. Les travaux de Peters $(1977,2001)$ sur les fillers, ces petits éléments d'abord indéterminés (niveau prémorphologique), qui se différencient progressivement selon la place qu'ils occupent dans un énoncé, devant un nom ou un verbe (niveau protomorphologique), avant de devenir des morphèmes à part entière, déterminants ou pronoms (niveau morphologique), montrent bien comment l'habileté croissante de l'enfant à produire et manipuler 
ces petits éléments, difficiles à identifier parce que non saillants, abstraits et souvent plurifonctionnels (Tomasello, 2003) est le signe d'un progrès développemental, sur plusieurs fronts simultanés (phonologique, distributionnel, lexical, pragmatique et syntaxique). Pour Peters (2001), les enfants doivent construire leurs catégories grammaticales sur la base d'un apprentissage lent et progressif de l'information phonologique, distributionnelle et fonctionnelle contenue dans l'input.

\section{Comprendre le processus, déconstruire les constructions ?}

Lorsqu'un enfant se met à parler, il est souvent difficile, voire impossible, de comprendre comment il est parvenu à ce « résultat ». A-t-il reproduit des blocs entendus chez l'adulte ? A-t-il analysé les énoncés de l'adulte pour en tirer des règles? Les deux en même temps? Selon Bowerman (1985), lorsque les énoncés de l'enfant ne sont pas «remarquables » eu égard aux normes langagières de sa communauté linguistique, il est difficile de déduire la connaissance qui les sous-tend. En effet, un usage correct en surface peut être compatible soit avec une connaissance proche de celle d'un adulte, soit avec une compréhension reposant sur des représentations de «morceaux » non analysés ou seulement de manière partielle, correspondant à des structures internes complexes chez l'adulte. En outre, même lorsque les enfants semblent avoir analysé certains de ces «morceaux», c'est-à-dire lorsqu'ils utilisent les morphèmes comme des éléments indépendants, il est toujours possible de décrire de manières différentes leur connaissance structurelle, tout comme chez l'adulte.

\subsection{Les « erreurs » de l'enfant}

C'est pourquoi de nombreux chercheurs s'appuient sur les « erreurs », les formes « déviantes » produites par les enfants, pour tenter de comprendre ce processus de construction du langage. Dès 1879, Egger postule que ces « erreurs » ou «barbarismes » sont des manifestations de «l'instinct grammatical» de l'enfant. Les études développementales se fondent souvent sur l'analyse d'erreurs (cf. Slobin 2001, pour les études fondées sur la comparaison de langues). Clark (2001) a développé la notion de «catégories émergentes » pour désigner certaines créations linguistiques de l'enfant, qui grammaticalise des catégories conceptuelles non exprimées dans sa langue maternelle (mais qui peuvent l'être dans d'autres langues). L'identification et l'analyse de ces catégories émergentes permet d'appréhender le rôle des universaux du langage dans l'acquisition, rôle mis en lumière par les tentatives de l'enfant de construire, de cartographier les catégories conceptuelles qui sont saillantes pour lui. D'autre part, l'enfant utilise souvent un élément lexical unique ou une construction unique pour exprimer tous les exemplaires d'une catégorie qu'il a identifiée. Ce phénomène de surextension le conduit à des erreurs systématiques, que ce soit au niveau lexical ou au niveau morphosyntaxique. C'est l'input qui fournira à l'enfant les moyens de modifier ses représentations et de corriger ses erreurs, ce qui peut être facilité par les reformulations ou rectifications par l'adulte des énoncés « déviants » de l'enfant (cf. Chouinard \& Clark 2000, Tomasello 2003, Leroy, Morgenstern \& Caët sous presse). Ainsi, au fur et à mesure de son développement, l'enfant passerait par différents stades, réajustant progressivement ses hypothèses sur la structure de la langue. Le langage de l'enfant se définirait ainsi comme un ensemble de systèmes qui se réorganisent de manière successive (Veneziano \& Sinclair, 2000). Mais le chemin est semé d'embûches, et certains emprunteront des impasses («blind alleys», Kilani-Schoch et al., 1997). Nous proposons ici, à l'aide d'une étude de cas, de tenter de comprendre le développement de certains marqueurs morphologiques, en partant d' « écarts » de l'enfant, que nous considérerons comme marques de son processus de construction de la langue.

\subsection{Une étude de corpus}

L'enfant étudiée ici, une petite fille française, Anaé, est filmée depuis sa naissance, une heure par mois, et ses productions vocales/verbales et gestuelles sont transcrites à l'aide du logiciel Clan, sous le format Chat (MacWhinney, 2000), dans le cadre du projet COLAJE financé par l'ANR (Projet ANR-08-COMO21 COLAJE Communication Langagière chez le jeune enfant, dirigé par Aliyah Morgenstern, 
http://colaje.risc.cnrs.fr/ ) et du projet ENFLANG, soutenu par le TGE ADONIS (http://www.tgeadonis.fr/?ENFLANG-base-de-donnees-sur-le ). Elle est filmée à son domicile, en situation d'interaction avec sa mère, et parfois ses frères aînés et/ou son père. Nous nous intéresserons ici à la période de son « explosion » grammaticale, à savoir entre 18 mois et 3 ans, âge de son entrée à l'école maternelle. Nous procéderons dans cet article à une analyse longitudinale de certains phénomènes remarquables dans le langage de cette enfant - les erreurs de genre sur les articles et l'expression de la possession - afin de tenter de comprendre la ou les stratégie(s) qu'elle utilise (globale et/ou analytique), dans son processus de construction de la grammaire.

\section{La question du genre}

En français, le genre, comme le nombre, se marquent sur le suffixe du nom et sur le déterminant précédant obligatoirement le nom. Le genre n'est pas nécessairement « visible» sur le nom mais toujours sur le déterminant, au singulier, qu'il s'agisse d'un article, défini (le, la) ou indéfini (un, une), d'un adjectif possessif (mon, ma, ton, ta, son, sa), démonstratif (ce, cette) ou indéfini (certain, certaine, chacun, chacune...). La marque de genre disparaît toutefois devant les noms commençant par une voyelle (l'article défini s'élide devant voyelle, et les adjectifs possessifs sont neutralisés sous la forme masculine mon, ton, son). Pour certains noms, le genre est déterminé par le sexe du référent (on parle de genre conceptuel), mais pour une quantité importante de noms, le genre est fixé de manière arbitraire par la langue (on parle dans ce cas de genre grammatical). Par conséquent, rien ne permet de déterminer/deviner le genre de ces unités, sauf le déterminant qui les précède, ce qui rend l'acquisition du genre difficile aux locuteurs non natifs. D'après Rondal et Seron (2000), les articles indéfinis apparaitraient aux alentours de 30 mois chez l'enfant et s'accorderaient en genre avec le nom déterminé vers 36 mois, âge d'apparition des articles définis. Le marquage grammatical du genre serait donc acquis avant celui du nombre (42 mois, d'après Rondal et Seron, 2000). Comment les enfants acquièrent-ils le genre grammatical en français? Les études précédentes portant sur des enfants monolingues apprenant l'italien ou le français montrent qu'aux alentours de 2 ans, lorsque les enfants commencent à produire les articles de la langue adulte, les erreurs d'accord de genre sont très peu nombreuses (seulement $1,5 \%$ d'erreurs entre $2 ; 01$ et $2 ; 05$ par exemple pour un enfant français étudié par Kupisch, Müller et Cantone (2002, cité par Roulet et al., 2004). Une étude sur le portugais brésilien citée par Roulet et al. (2004) montre que les enfants de moins de deux ans sont sensibles à la discordance de genre entre déterminant et nom, ce qui suggère selon les auteurs que «l'accord peut faire l'objet d'un calcul automatique et irrépressible même avant que les déterminants ne soient systématiquement produits par l'enfant». Karmiloff-Smith (1979) a montré que les enfants de trois ans, en situation expérimentale, sont sensibles à la régularité des marques du genre en français. Lorsqu'un mot fictif présente une terminaison ayant une forte probabilité de se situer sur un nom masculin, le déterminant choisi est masculin. Avant cinq ans, il semblerait que les enfants préfèrent l'information morpho-phonologique (véhiculée par la terminaison du mot) à l'information conceptuelle (sexe du référent) pour attribuer le genre. Pour Franck (2001), l'apprentissage de la catégorie syntaxique de genre s'effectue sur la base d'information formelle de nature probabiliste. Une autre façon de voir les choses serait d'envisager le déterminant, sur lequel se marque le genre du nom, comme étant acquis en même temps que le nom par l'enfant, comme une sorte de préfixe attaché au nom. Si l'enfant utilise une stratégie globale ("gestalt») et apprend les structures grammaticales comme des blocs, le déterminant et le nom ne seraient pas dissociés et il n'y aurait par conséquent aucune erreur sur le genre du déterminant. Au contraire, dans une stratégie analytique, l'enfant apprendrait le déterminant et le nom comme deux entités autonomes, et serait davantage susceptible de produire des erreurs.

Dans notre corpus, nous avons pu observer des erreurs de genre sur les déterminants. Nous pensons que leur analyse détaillée est susceptible de nous éclairer sur les mécanismes de mise en place du genre et sur le processus d'acquisition du syntagme nominal. Les graphiques 1 et 2 ci-dessous présentent le nombre d'occurrences d'articles définis et indéfinis dont le genre est correct (en blanc) par rapport aux formes incorrectes (en noir), dans chaque séance mensuelle d'une heure d'enregistrement. Les autres 
Psycholinguistique et acquisition

DOI $10.1051 / \mathrm{cmlf} / 2010111$

déterminants (adjectifs possessifs et démonstratifs, notamment) seront étudiés dans une recherche ultérieure.

\section{Articles définis}

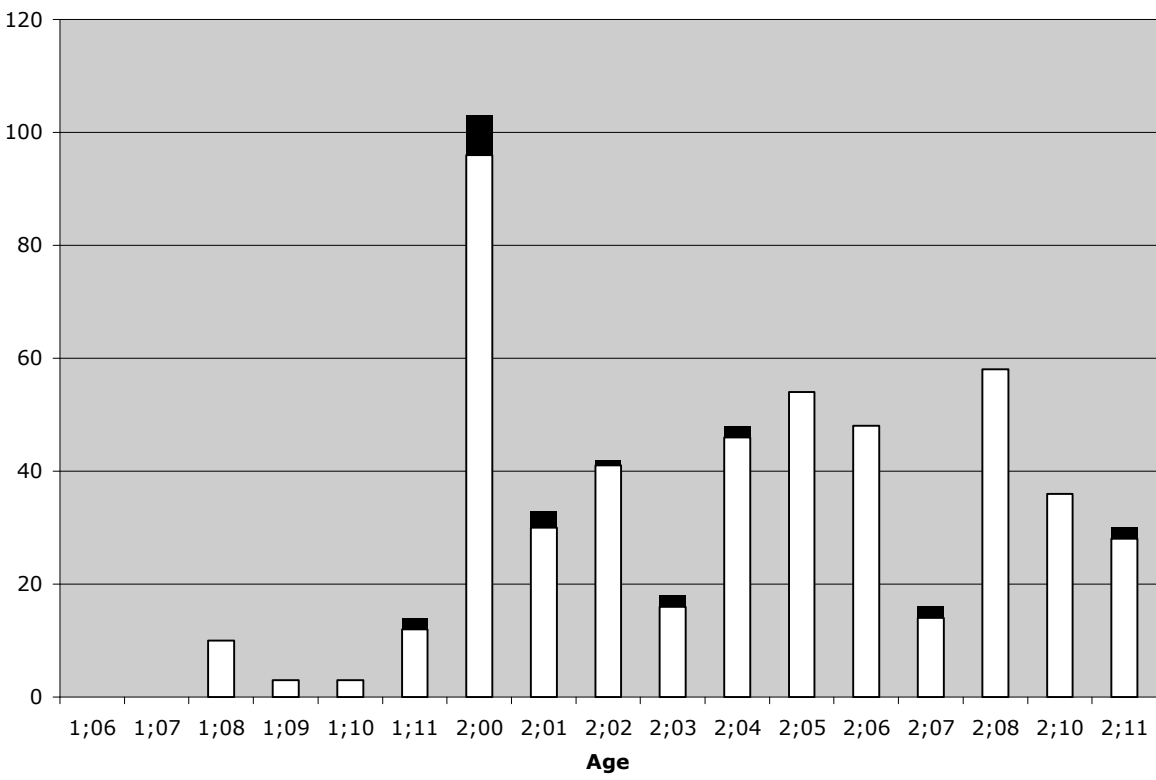

Graphique 1 : le genre des articles définis entre 1;06 et 2;11

\section{Articles indéfinis}

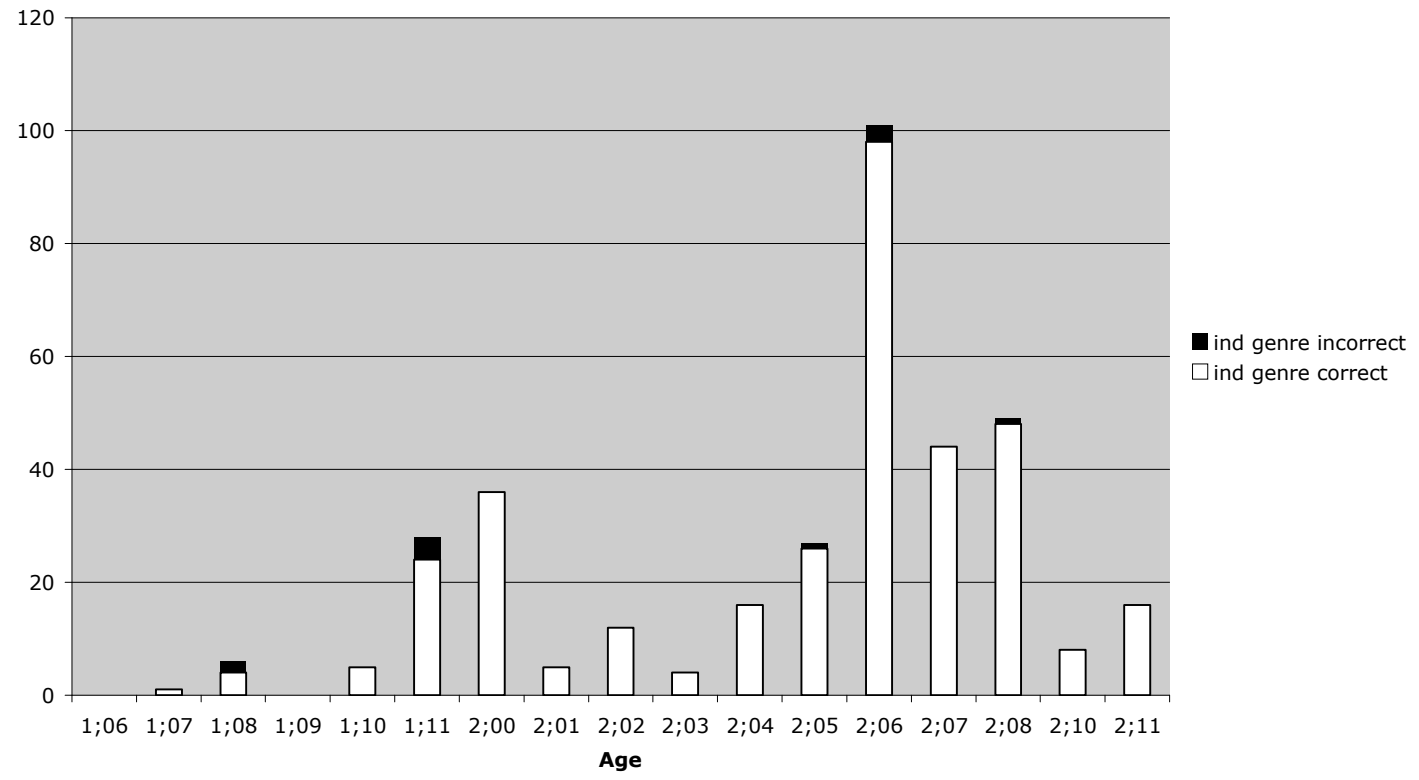


Les articles, définis et indéfinis, apparaissent à 1;08 dans notre corpus (en concurrence avec des « fillers » (cf. Peters, 2001 ; Veneziano \& Sinclair, 2000)), avec une augmentation importante à partir de 1;11. Le nombre d'occurrences dans la suite du corpus est fortement dépendant du contexte, mais la proportion d'erreurs sur le genre reste assez constante, entre 0 et $14 \%$, avec un maximum de $14 \%$ d'erreurs à $1 ; 11$. A 2;11, la proportion d'erreurs est encore de 5\%. Les erreurs de genre portent davantage sur les articles définis que sur les indéfinis. Le graphique 3 ci-dessous présente le pourcentage d'erreurs dans le corpus portant sur les articles définis et indéfinis confondus.

Pourcentage d'articles avec genre incorrect

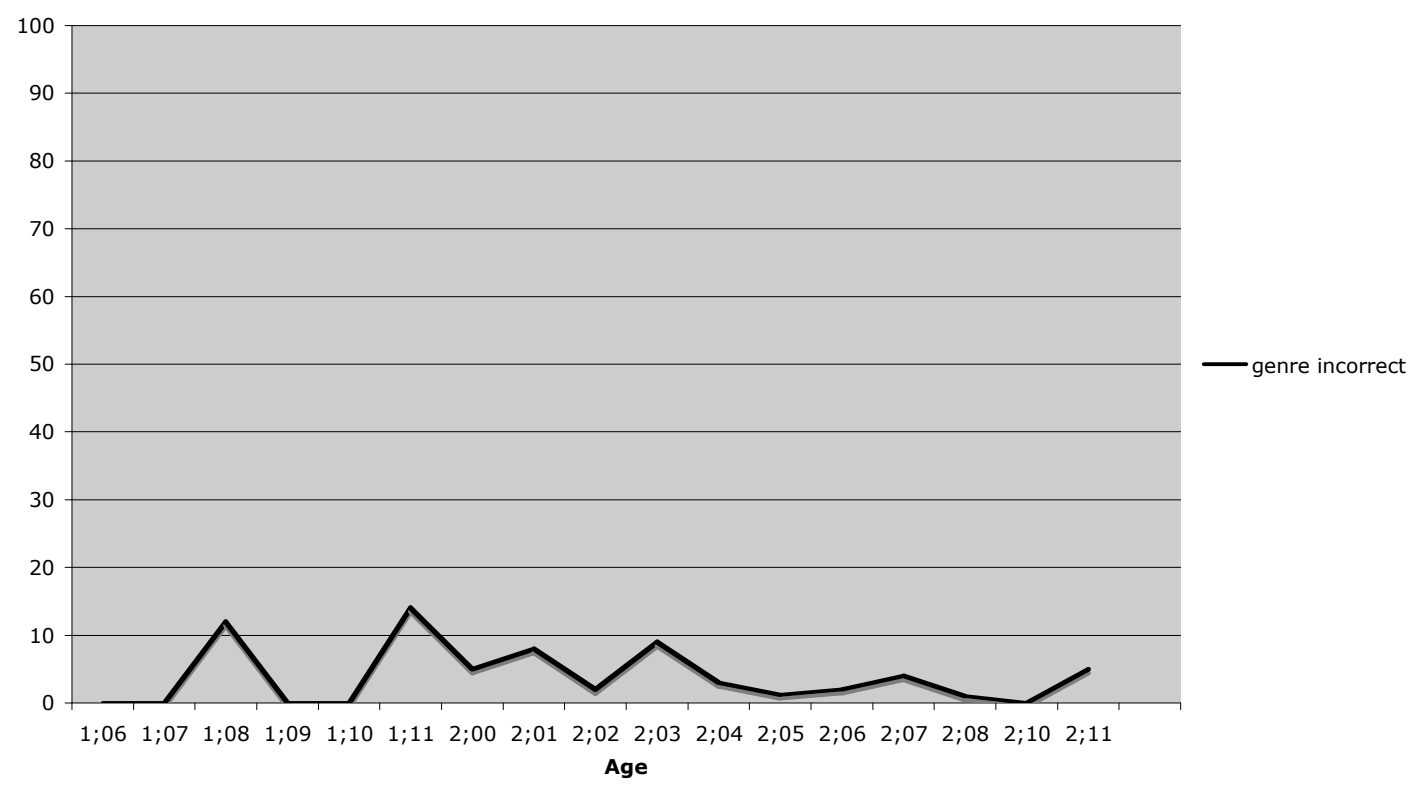

Graphique 3 : Pourcentage d'articles définis et indéfinis avec erreurs de genre

Il pourrait nous être objecté que les erreurs sur le genre des articles semblent anecdotiques dans le corpus, mais, même si elles sont peu nombreuses, elles n'en existent pas moins et méritent d'être prises en compte, dans la mesure où elles sont susceptibles de nous éclairer sur la façon dont l'enfant s'approprie la grammaire.

Le tableau ci-dessous donne des exemples d'erreurs de genre relevées dans le corpus:

\begin{tabular}{|c|l|l|}
\hline Age & Articles définis & Articles indéfinis \\
\hline $1 ; 08$ & la ballon & un pomme \\
\hline $1 ; 11$ & $\begin{array}{l}\text { le cuillère - la ballon - le poupée - le poule - le patate - la « palon » } \\
\text { (pantalon) }\end{array}$ & un bête - un fleur \\
\hline $2 ; 00$ & $\begin{array}{l}\text { le poupée - le « piquette (l'étiquette) - la couvercle - le pomme - } \\
\text { le fleur - la puzzle - la collier }\end{array}$ & \\
\hline $2 ; 01$ & le jambe - la couteau - la café & \\
\hline
\end{tabular}




\begin{tabular}{|c|l|l|}
\hline $2 ; 02$ & La [kœjœj] (l'écureuil) & \\
\hline $2 ; 03$ & Le bouteille - Le [lo] (l'eau) & \\
\hline $2 ; 04$ & La miel & \\
\hline $2 ; 05$ & & Un carte - un tête \\
\hline $2 ; 06$ & & Un fleur - un bouche - une tracteur \\
\hline $2 ; 07$ & Le caméra & \\
\hline $2 ; 08$ & & Un école \\
\hline $2 ; 09$ & & Une [RozwaR] (arrosoir) \\
\hline $2 ; 11$ & Le «nistoire » (l'histoire) & \\
\hline $3 ; 03$ & Le « zoreille» (l'oreille) - La «norteil» (l'orteil) & \\
\hline
\end{tabular}

Tableau 1 : Exemples d'erreurs de genre sur l'article

Une autre étude en cours, sur le même corpus, montre qu'à l'âge de 1;09, l'enfant produit encore très peu de déterminants. En effet, $70 \%$ des noms produits par l'enfant ne sont précédés d'aucun élément. 30\% des noms sont précédés d'un élément, dont seulement $13 \%$ sont des articles (la ou le). Les autres sont des éléments additionnels préfixés ou «fillers ». Au contraire, à l'âge de 2 ans, 93\% des noms produits par l'enfant sont précédés d'un élément. Parmi ces éléments préfixés au nom, près de $70 \%$ correspondent à un morphème grammatical (article ou autre déterminant), les 30\% restants présentent des formes instables et non conformes à une cible adulte (des « fillers »).

Comment expliquer les erreurs de genre et leur évolution? Si nous envisageons que les enfants construisent leurs propres systèmes de règles, à partir de stratégies plutôt analytiques ou plutôt globales, les erreurs sur le genre, assez fréquentes aux alentours de 1;11 - 2;00, âges auxquels les déterminants commencent à être utilisés de manière systématique devant les noms, révéleraient l'existence d'une stratégie plutôt analytique. En effet, l'enfant apprendrait les noms de manière isolée, repérerait que les noms doivent être précédés d'un déterminant, mais ne mémoriserait pas la suite déterminant + nom comme un bloc. La coexistence au sein d'une même séance de formes correctes et incorrectes (le ballon / la ballon, le pantalon / la pantalon) semble aller dans ce sens. Il est fort possible que certaines occurrences soient mémorisées directement avec le déterminant le plus fréquent dans l'input mais nous pouvons faire l'hypothèse d'un système transitoire entre le stade proto-morphologique et le stade morphologique, où l'enfant produirait des formes existant dans la langue adulte (des articles en l'occurrence) mais sans en maîtriser la ou les fonctions. En effet, l'article est à la fois un marqueur d'actualisation (défini ou indéfini) mais également un marqueur de genre et de nombre. D'après Hurtig et Rondal (1999), les indéfinis tendent à être employés en lieu et place des définis à 54 mois (4 ans $1 / 2)$, avec un emploi correct des articles seulement à 72 mois (à savoir 6 ans). Les articles s'accordent en genre avec le nom déterminé à 36 mois, et en nombre à 42 mois. Par conséquent, il est possible d'envisager l'article comme un amalgame de plusieurs fonctions que l'enfant va apprendre à utiliser et combiner progressivement. Si l'enfant ne mémorise pas directement le nom avec l'article adéquat, il lui est impossible de construire une règle lui permettant de retrouver l'article puisque le genre est arbitraire dans la plupart des cas. L'enfant utiliserait donc dans un premier temps les articles comme des « fillers », c'està-dire comme des éléments remplissant la place d'un marqueur morphologique obligatoire, mais pas encore associés à des fonctions, comme celle de marquer le genre du nom par exemple. Comme il existe plusieurs formes qu'il ne différencie pas encore, il les utiliserait de façon aléatoire. Petit à petit, en s'appuyant sur l'input, l'enfant mémoriserait de plus en plus de noms avec les déterminants correspondants, sans pour autant analyser ces derniers comme des unités signifiantes ou fonctionnelles. Il existerait donc plusieurs étapes ou sous-étapes dans le processus de construction du syntagme nominal : tout d'abord, le nom est produit seul, puis précédé d'un élément préfixé ( « filler » ou proto-déterminant, c'est-à-dire un élément dont la forme est celle d'un déterminant adulte, mais qui n'en possède pas encore la ou les fonctions), ce qui serait le signe d'une conscience du syntagme nominal comme une unité 
complexe, mais pas encore d'une capacité à analyser les fonctions (genre, nombre...) associées aux formes.

Nous pouvons illustrer cette hypothèse à partir de l'évolution des lexèmes "poule » et «fleur » dans le corpus (le nombre d'occurrences dans la séance d'enregistrement et les productions en répétition de l'adulte sont indiqués entre parenthèses) :

\begin{tabular}{|l|l|l|l|l|l|l|l|}
\hline Age & $1 ; 07$ & $1 ; 09$ & $2 ; 00$ & $2 ; 02$ & $2 ; 03$ & $2 ; 05$ & $2 ; 08$ \\
\hline $\begin{array}{l}\text { Production } \\
\text { de l'enfant }\end{array}$ & poule & $\begin{array}{l}{[\mathrm{i}] \text { poule }} \\
{[\varepsilon] \text { poule }} \\
\text { (x2) }\end{array}$ & $\begin{array}{l}\text { [a] poule } \\
\text { la poule }\end{array}$ & $\begin{array}{l}\text { La poule } \\
(\mathrm{x} 2)\end{array}$ & $\begin{array}{l}\text { Le poule } \\
(\mathrm{x} 2) \\
\text { La poule } \\
\text { (répétition) }\end{array}$ & $\begin{array}{l}\text { La poule } \\
\text { Une poule } \\
(\mathrm{x} 2)\end{array}$ & [y] poule \\
\hline
\end{tabular}

Tableau 2 : évolution du lexème « poule » dans le corpus

\begin{tabular}{|l|l|l|l|l|l|l|l|}
\hline Age & $1 ; 09$ & $1 ; 10$ & $2 ; 00$ & $2 ; 02$ & $2 ; 06$ & $2 ; 09$ & $2 ; 10$ \\
\hline $\begin{array}{l}\text { Production } \\
\text { de l'enfant }\end{array}$ & La fleur & Une fleur & La fleur & $\begin{array}{l}\text { Une fleur } \\
(\mathrm{x} 2) \\
\text { (répétition) }\end{array}$ & $\begin{array}{l}\text { Un fleur } \\
\text { Une fleur } \\
\text { (répétition) }\end{array}$ & La fleur & Une fleur \\
\hline
\end{tabular}

Tableau 3 : évolution du lexème « fleur » dans le corpus

L'input et les capacités de mémoire jouent vraisemblablement un rôle fondamental dans ce processus d'appropriation des déterminants par l'enfant. En effet, la marque de genre étant arbitraire, elle ne peut s'acquérir / trouver sa source que dans l'input, qui fournit à l'enfant les informations à mémoriser et stocker dans son lexique mental. Cependant, pour qu'il puisse réinvestir les données provenant de l'input, et notamment les informations portant sur le genre du nom véhiculées par le déterminant, et les appliquer dans d'autres contextes, l'enfant doit avoir repéré et analysé les formes (les morphèmes) et les fonctions qui y sont attachées.

Par conséquent, certaines erreurs sur le genre des articles pourraient s'expliquer par cette étape intermédiaire entre conscience de la nécessité d'un article devant le nom, et capacité d'analyse morphologique. Le faible nombre d'erreurs serait à mettre sur le compte des capacités de mémoire de l'enfant, et de la fréquence du mot-cible dans l'input.

On pourrait également expliquer les erreurs sur le genre des articles par d'autres facteurs, dont certains peuvent être concomitants :

- la fréquence : les noms peu fréquents dans l'input vont être produits avec un article choisi «au hasard », ou sur la base de propriétés morpho-phonologiques du nom (certains suffixes étant plus masculins que d'autres, cf. Karmiloff, 1979), ou encore par analogie avec des noms dont le déterminant est connu ;

- L'initiale du nom : lorsqu'un nom commence par une voyelle, l'article défini s'élide, et perd donc sa marque de genre. Si le nom apparaît généralement précédé d'un défini dans l'input, il est difficile pour l'enfant d'accéder à son genre. Nous trouvons plusieurs exemples dans le corpus : oreille ("le zoreille »), écureuil ("la [kojoj] »), eau ("le [lo] »), école (" un école »), arrosoir ("une [RozwaR]»), histoire ("le [nistwaR]»), orteil ("la norteil »). Nous pouvons observer que l'enfant semble privilégier le schéma syllabique CVCV et a tendance à rattacher au nom une consonne provenant d'un déterminant, soit indéfini ("noreille », "nistoire », "norteil »), soit pluriel (《zoreille »). Dans certains cas, la forme produite par l'enfant semble résulter d'une erreur de segmentation de l'input: [kœjoj] pour "l'écureuil », [RozwaR] pour "l'arrosoir », le [lo] pour «l'eau ». D'une part, l'enfant mémorise une forme erronée du nom, et d'autre part, il 
doit recalculer le déterminant, et va produire soit une forme aléatoire, soit une forme résultant de son erreur de segmentation. Ainsi, "l'arrosoir », segmenté en "la [RozwaR] 》 va entraîner la forme « une [RozwaR] », ce qui est le signe que l'enfant est capable à cet âge $(2 ; 09)$ de réinvestir ses connaissances morphologiques -ici, erronées- dans un autre contexte («la» devient « une »);

- Le nombre : certains noms sont souvent utilisés au pluriel : c'est le cas par exemple de jambe, carte, orteil, oreille, main, ou sont précédés d'un partitif (miel, eau), ce qui ne permet pas à l'enfant de déduire facilement le genre de ces noms en se fondant uniquement sur l'input.

- La proximité (sémantique et/ou syntaxique) de noms du genre opposé : par exemple, la couteau est produit par l'enfant juste après la fourchette. En ce qui concerne la café, l'enfant observée semble associer et même substituer le mot café au mot tasse, dans des énoncés comme " $j$ 'a [bwa] la café » au retour de la piscine, pour raconter qu'elle a bu la tasse.

Le genre des noms étant fixé arbitrairement par la langue, l'enfant se retrouve dans l'incapacité de construire une règle lui permettant de recalculer automatiquement les formes qu'il n'a pas mémorisées (une sorte de règle par défaut, permettant de produire des énoncés n'ayant jamais été entendus auparavant). Contrairement à la morphologie verbale par exemple, qui permet d'établir des règles par défaut, du type « ajout de $[\varepsilon]$ » au verbe pour une action passée, sur le modèle de « chante / chantais », ce qui entraîne des erreurs comme « ils sontaient» pour «ils étaient» pour les verbes irréguliers (qui, par définition, échappent à la règle et ne peuvent être recalculés automatiquement), le genre ne peut pas faire l'objet d'une règle valable pour tous les noms. En outre, contrairement aux marques de temps, le genre ne peut être associé à une intention communicative ou pragmatique particulière. Il est plus facile pour l'enfant d'associer le morphème $[\varepsilon]$ à sa valeur de " passé », que d'associer la marque de genre à un sens ou une fonction. Par conséquent, l'aspect purement formel du genre au sein du système rend son acquisition complexe.

Un autre élément mérite d'être pris en compte pour traiter la question du genre : les pronoms personnels de $3{ }^{\text {ème }}$ personne (il et elle), qui apparaissent selon Hurtig et Rondal (1999) à 36 mois, au même âge que l'accord de l'article en genre avec le nom déterminé, semblent suivre le même processus. Même si on observe des formes pronominales différenciées devant les verbes, leur genre semble souvent aléatoire dans un premier temps. On peut citer des exemples d'énoncés où le genre du nom, du pronom et/ou de l'adjectif ne sont pas cohérents : Il est coquine Omer (nom du chien de la maison) (1;11), La luge il est là (2;00), La valise il est beau à moi; elle est mignon ce chien (2;02), Elle est assis kirikou (2;08), elle est vert le dinosaure $(2 ; 11)$. Ces exemples semblent aller dans le sens d'un traitement des morphèmes grammaticaux (articles et pronoms) comme des marqueurs obligatoires encadrant des unités signifiantes (noms, verbes et adjectifs) mais pas encore associés à des concepts abstraits comme le genre. Ainsi, les enfants ne deviendraient conscients des propriétés phonologiques, morphologiques et syntaxiques en rapport avec le genre que très tard dans leur développement (Karmiloff-Smith, 1979). Au contraire, lorsque l'enfant commence à analyser les morphèmes grammaticaux comme des unités signifiantes, il va avoir tendance à surgénéraliser certains marqueurs (Clark, 2001). Ainsi, à l'âge où apparaissent les surgénéralisations au niveau de la morphologie verbale, du type «ils sontaient» pour «ils étaient», ou encore «je dorra » pour « je dormirai », aux alentours de trois ans, on observe le même phénomène au niveau des adjectifs, voire des adverbes, pour marquer le genre féminin. On peut citer par exemple dans notre corpus " ma robe elle est jolisse » (à 2;09) ou " ma chaussette elle est pas à l'enverte " (à 3;03). Ce type d'erreurs montre que l'enfant a identifié certains petits éléments (ici, les marques de genre) et qu'il leur assigne un «sens », une valeur ou fonction dans le système, ce qui ne semblait pas être le cas précédemment. Par conséquent, même si, selon Brown (1973), l'ordre d'acquisition des morphèmes peut s'expliquer en faisant appel à la complexité sémantique, c'est-à-dire le nombre de significations exprimées par le morphème, et/ou syntaxique (le nombre de règles requises par le morphème en question), il est nécessaire, à l'instar de Hudelot et Salazar (2004), « de rappeler que le fait de commencer à utiliser certains outils grammaticaux n'implique pas nécessairement que l'enfant maîtrise pleinement leur fonction ». Il y aurait donc plusieurs étapes dans l'acquisition de la morphologie, notamment pour les marqueurs complexes, comme cela semble être le cas des déterminants, qui cumulent plusieurs fonctions. 
L'apparition d'articles ou de pronoms différenciés, notamment au niveau du genre, ne garantit pas que l'enfant maîtrise pleinement leur fonctionnement.

Pour conclure sur la question $\mathrm{du}$ genre, nous pouvons établir un parallèle avec l'hypothèse développementale de la «courbe en $U$ » : dans un premier temps, les enfants utiliseraient les formes correctes comme s'il s'agissait d'items indépendants, fixes. Puis il découvriraient les règles et les appliqueraient de manière indifférenciée à tous les mots concernés (phase de surgénéralisation), avant de revenir à une utilisation correcte, une fois qu'ils auraient intégré les règles et leurs exceptions (Karmiloff \& Karmiloff-Smith, 2001). L'exemple souvent cité est celui du morphème de passé -ed en anglais : l'enfant commencerait par produire la forme correcte went pour le prétérit irrégulier de to go, puis surgénéraliserait la règle verbe + -ed à tous les verbes, y compris les irréguliers, d'où la forme goed, avant de revenir à la forme correcte went. Selon Marcus \& al. (1992), on peut expliquer ce phénomène de la manière suivante : les formes irrégulières seraient apprises par cœur par l'enfant, alors que les formes régulières feraient l'objet de l'établissement d'une règle. La règle pour les verbes réguliers serait par conséquent la règle par défaut. Il y aurait donc une asymétrie entre les verbes réguliers et les verbes irréguliers. La formation des verbes réguliers relevant d'une règle par défaut, l'enfant n'aurait pas besoin d'entendre ces verbes une seule fois pour être capable de les construire. Les verbes irréguliers, au contraire, échappent par définition à la règle, et, ne pouvant être « reconstruits », doivent donc être appris par cœur.

En ce qui concerne le genre, le tableau développemental pourrait être le suivant :

- les premières occurrences de syntagmes nominaux composés d'un article et d'un nom seraient appris comme des blocs, des structures figées. Ainsi, à $1 ; 09$, lorsqu'Anaé produit le syntagme «la fleur », elle produit dans la même séance les séquences figées [tõbebe] pour désigner sa poupée, et [tatzt] pour désigner la tête d'une grenouille. On peut donc supposer que la suite « la fleur » constitue pour elle un seul bloc.

- Dans un second temps, l'enfant analyserait les deux éléments du syntagme comme deux unités distinctes (déterminant + nom), et en déduirait une proto-règle selon laquelle tout nom doit être précédé d'un déterminant. Ainsi, de la même manière qu'un petit anglais va produire " goed » au lieu de «went », surgénéralisant ainsi la règle par défaut de formation du passé, le petit français va appliquer la règle du déterminant obligatoire en position pré-nominale. Cependant, la conscience de la nécessité d'un élément avant le nom ne signifie pas la maîtrise de sa ou ses formes et/ou fonctions. On trouvera alors en position pré-nominale des éléments à la forme incertaine (des «fillers ») ou des articles bien formés au niveau phonologique, proches de la forme adulte, mais parfois choisis de manière aléatoire dans le paradigme à disposition de l'enfant. Ainsi, on aurait pendant cette période une sorte de va-et-vient entre une stratégie globale (avec des syntagmes nominaux produits comme des blocs dans lesquels le déterminant et le nom ne sont pas différenciés) et une stratégie analytique, avec une tentative de construction d'une règle pour le genre des déterminants.

- Dans un troisième temps, dans l'hypothèse de la « courbe en U », l'enfant analyse les formes et leurs fonctions, établit et applique des règles, tout en mémorisant les exceptions. En ce qui concerne le genre, nous pourrions dire que, dans la mesure où il est arbitraire, toutes les formes fonctionnent comme des "exceptions», aucune règle ne pouvant être établie par défaut. L'enfant doit donc obligatoirement mémoriser le genre des noms, à partir des informations fournies par l'input, notamment les informations contenues dans les déterminants précédant les noms.

- Lorsque l'enfant est capable de réinvestir ces informations dans d'autres contextes, c'est-à-dire d'appliquer les marques de genre d'un nom à tous les déterminants susceptibles de le précéder, mais également aux adjectifs et aux pronoms, on peut dire qu'il a intégré la notion de genre, aussi abstraite soit-elle, et qu'il a établi une nouvelle règle selon laquelle tous les noms ont un genre qui se marque sur le déterminant et éventuellement sur d'autres éléments comme l'adjectif. 
Par conséquent, l'appropriation par l'enfant de certains marqueurs complexes dans la langue serait progressive et transiterait par des sous-systèmes de règles, tant au niveau de la forme, de la structure (par exemple, tout nom doit être précédé d'un déterminant) que de la ou des fonctions (par exemple, tout nom a un genre, marqué sur le déterminant).

L'analyse de l'acquisition des déterminants possessifs nous semble aller dans le même sens et pouvoir confirmer cette hypothèse.

\section{Les adjectifs possessifs}

De la même manière que l'article, l'adjectif possessif semble pouvoir être analysé comme un amalgame de plusieurs fonctions, ce qui en ferait un marqueur simple en apparence, au niveau syntaxique (en surface), mais complexe au niveau sémantique. Ainsi, selon Rondal et Seron (2000), l'emploi des adjectifs possessifs répond à un principe d'économie qui consiste à ramener les expressions du type « $\mathrm{X}$ appartient à $\mathrm{Y}$ » ou « $\mathrm{X}$ fait partie de $\mathrm{Y}$ » aux expressions du type «Adjectif possessif $+\mathrm{X}$ », et la sélection des adjectifs possessifs correspond à trois opérations : (1) la sélection de la personne en fonction du contexte interpersonnel de l'interaction linguistique (P1 quand le locuteur est le possédant, P2 quand le récepteur est le possédant, et P3 quand le possédant n'est ni le locuteur ni le récepteur), (2) la sélection de la personne selon le genre et le nombre du possédé (seulement au singulier pour le genre en français : $m o n / m a$ ) et (3) la sélection selon le nombre du possédant (singulier ou pluriel). Dans un premier temps, selon ces auteurs, l'enfant n'utilise pas l'adjectif possessif mais exprime la possession de trois façons :

- (préposition « à ») + possédant : (à) moi

- $\quad$ (article) + possédé + (préposition à/de) + possédant : la balle de Dédé

- possédant + possédé : papa bic

Les trois graphiques ci-dessous présentent les procédés pour marquer la possession à la $1^{\text {ìre }}$ personne du singulier (le locuteur), la $2^{\text {ème }}$ personne (le récepteur) et la $3^{\text {ème }}$ personne (extérieure à la situation d'interlocution) dans notre corpus, entre 1;06 et 2;11, pour chaque séance d'une heure d'enregistrement.

Possession P1

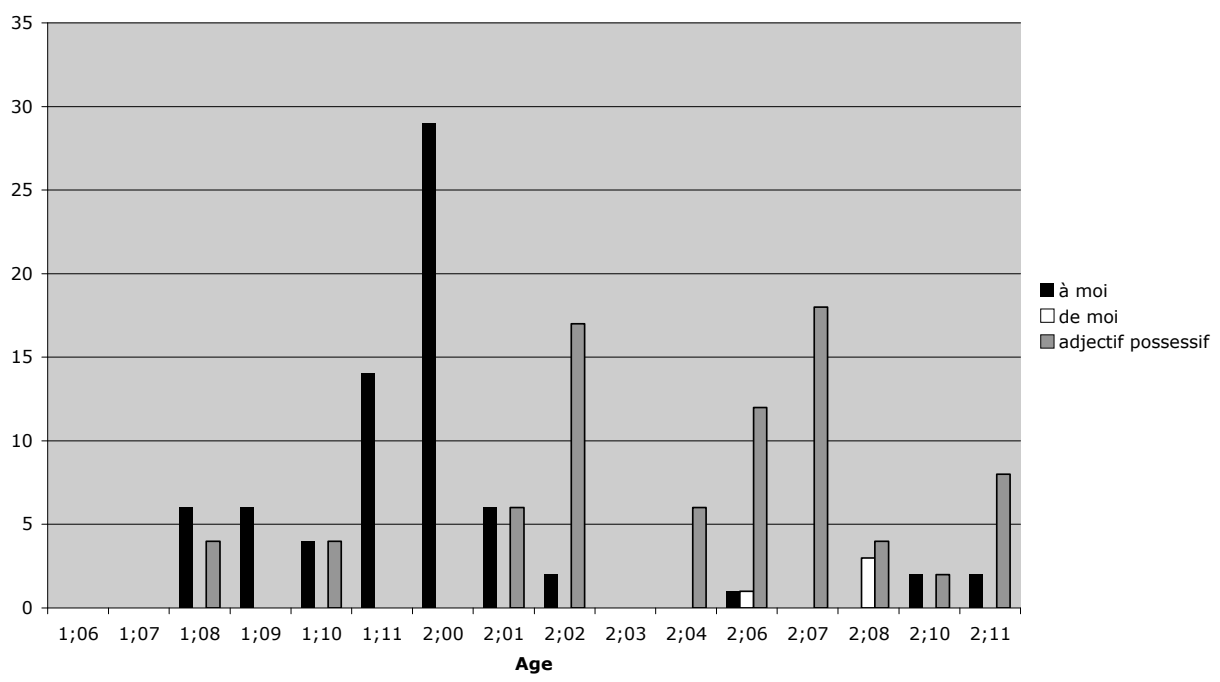

Graphique 4 : Expression de la possession à la $1^{\text {ère }}$ personne du singulier 


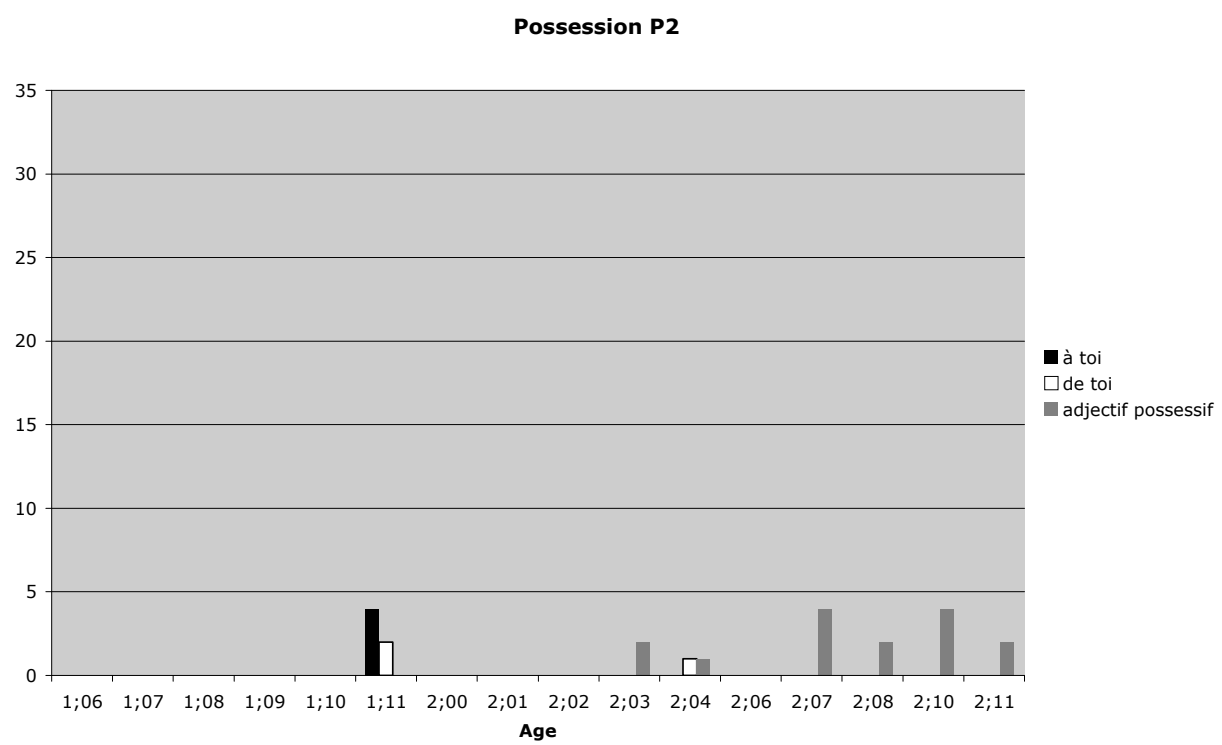

Graphique 5 : Expression de la possession à la $2^{\text {ème }}$ personne du singulier

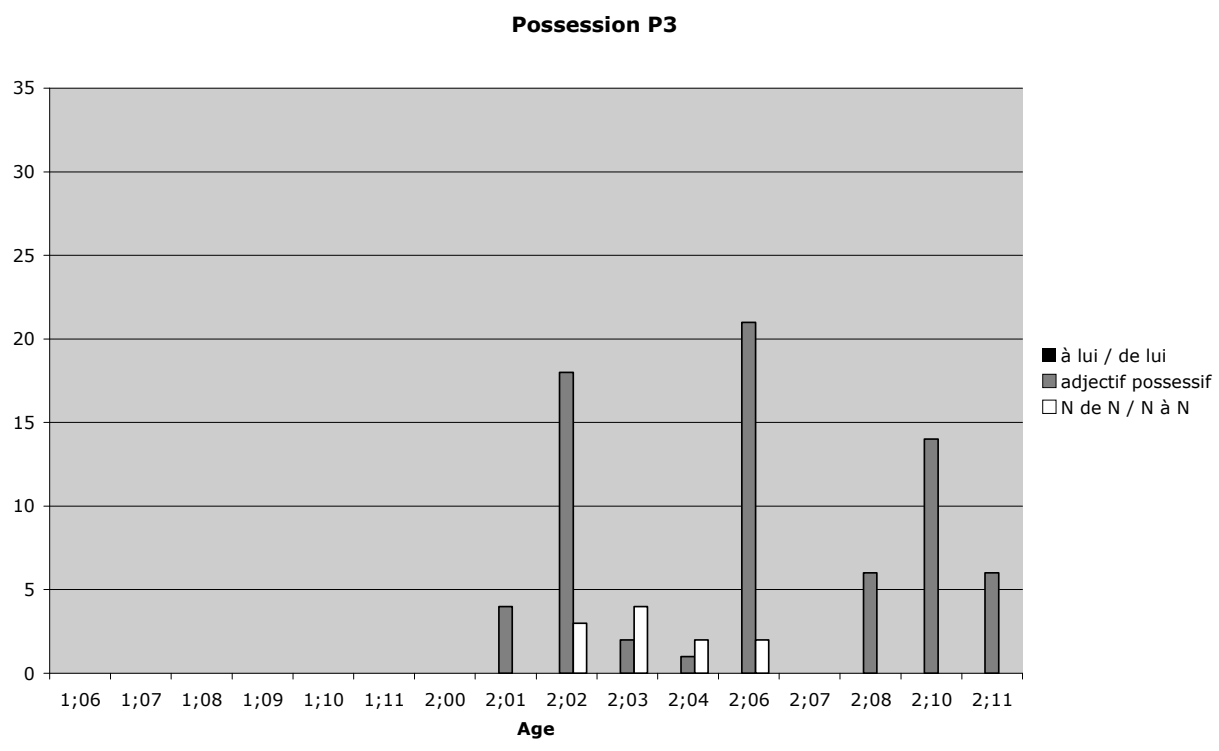

Graphique 6 : Expression de la possession à la $3^{\text {ème }}$ personne du singulier

Nous pouvons remarquer que la possession se marque plus tôt et de manière beaucoup plus fréquente à la première personne, c'est-à-dire par rapport à l'enfant lui-même. Dans un premier temps, la possession se marque essentiellement sous la forme «à moi », ou "c'est à moi », parfois accompagnée du déictique «ça » $((c ̧ a)$ c'est à moi $(c ̧ a))$. Pour Morgenstern et Sekali (1997), la préposition «à » dans « à moi » permet de redéfinir une propriété de l'objet dans un contexte conflictuel. L'enfant marque la possession d'un élément présent dans la situation, en effectuant une opération de repérage de cet élément par rapport à lui-même. Cette opération permet, selon Danon-Boileau et Brigaudiot (1996), d'exprimer «l'identité d'un objet en le rapportant de manière singulière à un individu extérieur à lui-même ». A partir de $1 ; 11$, dans notre corpus, cette même forme est utilisée dans la tournure: «le $\mathrm{N}$ à moi ». Selon Chaudenson (2004), avec le pronom personnel postposé, l'enfant construit sa propre règle à partir d'énoncés où 
figurent non pas des pronoms personnels mais des substantifs. En effet, ces constructions postposées ne se trouvent jamais dans l'input : l'enfant déduirait donc la construction « $\mathrm{X}$ de/à moi » à la fois de la construction possessive avec un substantif («le $\mathrm{X}$ de $\mathrm{Y} »)$ et du tour «c'est à moi ».

Dans notre corpus, l'enfant semble marquer la relation de possession ou encore le destinataire d'une action par cette tournure «à moi », dans des constructions originales, non présentes dans l'input. Ainsi, à 2;00, à côté d'énoncés comme " $c$ 'est le puzzle à moi », on trouve les énoncés " elle m'embête à moi » ou 《le loup a pas mangé à moi». Au même âge, la préposition «à » commence à être utilisée pour marquer la possession concernant d'autres personnes que l'enfant lui-même ("c'est à maman ", "pieds à Kirikou», "la tête à Kirikou»), même si sa valeur la plus fréquente est une valeur de localisation/destination (" cognée à canapé 》, " elle va à la douche »). La possession à la $2^{\text {ème }}$ personne du singulier est très peu marquée, mais apparaît sous la forme «à toi » et « de toi » à 1;11 («ce verre à toi », «un verre de toi »). Le fait que la $2^{\text {ème }}$ personne, de même que la $3^{\text {ème }}$ personne, soient très peu marquées et de manière plus tardive, peut être vu comme un effet de contexte (les situations d'enregistrement se prêtant davantage à l'expression de la possession à la $1^{\text {ère }}$ personne), ou comme la conséquence d'une difficulté à effectuer un repérage de l'objet par rapport à l'autre. La tournure « $\mathrm{N}$ de moi » est fréquente chez Eva, étudiée par Sourdot (1977), qui note que le sens d'appartenance est toujours rendu $\mathrm{par} / \mathrm{d} / \mathrm{par}$ cette enfant là où l'adulte a le choix entre plusieurs procédés (et notamment l'utilisation de l'adjectif possessif). L'enfant adopte et étend la structure la plus générale ( $\mathrm{X}$ de $\mathrm{Y}$ ), utilisant ainsi au mieux les possibilités de systématisation dont il dispose, sans toutefois prendre en compte les contraintes formelles (et notamment le fait que la tournure « $\mathrm{N}$ de moi» ne soit pas attestée dans la langue). Il est intéressant de noter que, dans notre corpus, cette tournure « $\mathrm{N}$ de moi / de toi » est plus tardive et beaucoup moins fréquente que la tournure « $\mathrm{N}$ à moi / à toi », bien que cette dernière tournure ne puisse être déduite de l'input (la possession étant généralement marquée par la préposition «de » en français contemporain, du moins dans le milieu socio-culturel dans lequel évolue l'enfant observée). L'enfant semble donc construire son propre système pour marquer la relation de possession, notamment celle qui le concerne directement, à partir du tour «à moi » vraisemblablement traité comme un bloc figé. On trouve d'ailleurs chez certains enfants la construction «le $N$ de à moi». Les adjectifs possessifs apparaissent à 2;02 (on trouve de rares occurrences à 1;08 et 1;10 mais qui semblent devoir être considérées comme des tournures figées, des blocs), sous des formes variées en genre, nombre et personne (mon, ma, mes, ton, ta, tes, son, sa, ses), qui remplacent la tournure « $\mathrm{N}$ à moi » qui disparaît alors complètement. A 2;08, on observe une tournure «complexe » qui mérite à notre avis un intérêt particulier : " mon truc de moi ». Si l'on envisage l'adjectif possessif comme un amalgame complexe de marqueurs à la fois d'une relation de possession et de marques de personne, de genre et de nombre, ce type de tournure «mon $\mathrm{N}$ de moi » peut être certes interprété comme une emphase sur la relation de possession, mais également comme la preuve que l'adjectif possessif n'est pas encore envisagé par l'enfant dans toute sa complexité sémantique, c'est-à-dire notamment comme un marqueur de possession. Le fait que l'enfant commence à marquer la possession par une tournure qui expose en quelque sorte la structure interne de l'adjectif possessif ( $"$ mon $N$ » peut être glosé par «le $N$ de moi») serait une des manifestations d'une capacité précoce de l'enfant à utiliser des «petits morceaux » pour construire des unités signifiantes, même si non conformes à la grammaire de la langue (Tomasello, 2003). De la même manière que pour l'article, à partir du moment où l'enfant est capable de faire varier la forme de l'adjectif possessif dans un même contexte, en fonction du possédant (P1, P2 ou P3) par exemple, on peut supposer qu'il a accédé à un certain niveau de connaissance de la relation forme/fonction de ce marqueur complexe. Ainsi, à 3;08, Anaé se livre à une opération de commutation révélatrice de ses connaissances (peut-être encore partielles) du fonctionnement des adjectifs possessifs, mais à partir d'une segmentation erronée :

Anaé, son frère Ael et leur mère parlent de leur grand-père de Montpellier.

Mère - c'est le papy de Montpellier

Anaé - le papy de [tõpolje]

Frère - non, le papy de Montpellier

Anaé - Maman, Ael il dit que c'est le papy de [sõpəlje] mais c'est le papy de [tõpalje]. 


\section{Conclusion}

L'analyse de la mise en place de la morphologie chez un enfant français, à partir de la marque de genre du déterminant et de l'expression de la possession, semble aller dans le sens des théories exposées en première partie de cet article, à savoir l'acquisition de la grammaire par constructions de l'enfant, assemblages de petits morceaux dans des structures, sans toutefois accéder immédiatement à la complexité de chaque marqueur. Nous avons pu observer, dans notre corpus, notamment à travers les « erreurs » de l'enfant, les traces d'une analyse de la langue, d'un processus de construction progressif, et en même temps d'un va-et-vient entre deux "stratégies ", à la fois globale, l'enfant repérant et utilisant des blocs sans les analyser (article + nom par exemple, ou le bloc « à moi ») et analytique. La fréquence extrêmement élevée des formes correctes par rapport aux erreurs s'explique en grande partie par le rôle du langage entendu et réutilisé et par les capacités de mémorisation des enfants, même si certains enfants, comme celui de notre corpus, ne semblent pas s'en contenter et ont tendance à " grammaticaliser » leurs productions, en « réinventant» la langue de manière créative. L'enfant analyse le langage qu'il entend, émet des hypothèses sur son fonctionnement, établit des règles et les « teste » en dialogue avec l'adulte. $\mathrm{Au}$ fur et à mesure des interactions, l'adulte soutient l'enfant dans cet apprentissage, en reformulant ses énoncés erronés, l'amenant progressivement à affiner ses hypothèses (cf. Chouinard \& Clark, 2000, et Leroy, Morgenstern, Caët, sous presse). L'acquisition du langage relève d'un processus complexe, consistant à être capable de combiner, de faire interagir une quantité importante d'éléments, aux niveaux phonologique, morphologique, syntaxique, lexical, pragmatique (cf. Leroy \& Martel, sous presse). L'analyse fine de corpus longitudinaux, et notamment des erreurs produites par les enfants, nous permet d'établir que ce n'est pas parce qu'on produit une forme qu'on en maîtrise nécessairement la ou les fonctions (cf. la question du genre traitée dans cet article), et que ce n'est pas parce qu'on est capable de marquer une fonction (ou d'exprimer un concept) qu'on en maittrise pour autant les formes linguistiques conventionnelles (cf. la question de la possession). L'objectif de l'enfant, dans son appropriation de la langue, sera de faire coïncider formes et fonctions, unités linguistiques et concepts, ou encore signifiants et signifiés. Une analyse approfondie des autres marqueurs morphologiques dans le corpus d'Anaé (et notamment de la mise en place de la morphologie verbale) nous permettrait de confirmer ces hypothèses et d'avoir une vue globale du processus d'acquisition de la morphologie chez cette enfant.

\section{Références bibliographiques}

Bassano, D. (2005). Développement du lexique et émergence de la grammaire. Le langage et l'homme, XXXX.2, 722.

Bloom, L. (1970). Language development : form and function in emerging grammars, Cambridge MA : MIT Press.

Bowerman, M. (1985). What shapes Children's Grammars?. In Slobin, D. (éd.), The crosslinguistic study of language acquisition, vol.2 : Theoretical issues. Hillsdale, NJ : Lawrence Erlbaum, 1257-1319.

Brown, R. (1973). A first language : The early stages. MA : Harvard University Press.

Clark, E. (2001). Emergent categories. In Bowerman, M. \& Levinson, S.C. (éd.), Language acquisition and conceptual development, Cambridge University Press (réédition 2003).

Chaudenson, R. (2004). La créolisation : théorie, applications, implications. Paris : L’Harmattan.

Chouinard, M.M. \& Clark, E.V. (2000). Enoncés enfantins et reformulations adultes dans l'acquisition du langage. Langages, vol.34, 140, 9-23.

Danon-Boileau, L., Brigaudiot, M. (1996). La relation d'appartenance : évolution de ses valeurs chez l'enfant. Faits de langues, vol.4, 7, 63-70.

Dressler, W. (1995). The theoretical relevance of pre- and protomorphology in language acquisition. Language, 81, 882-906.

Dressler, W., Kilani-Schoch, M., Klampfer, S. (2003). How does a child detect morphology? Evidence from production. In Bisang, W., Hock, H.H., Winter, W. (éd.), Trends in Linguistics, Studies and Monographs, 151, Mouton de Gruyter, 391-425. 
Egger, M.E. (1879). Observations et réflexions sur le développement de l'intelligence et du langage chez l'enfant. Paris : Picard.

Franck, J. (2001). Le genre : aspects théoriques et pratiques. Les Cahiers de la $S B L U, n^{\circ} 7 / 8,15-20$.

François, F., François, D., Sabeau-Jouannet, E., Sourdot, M. (1977). La syntaxe de l'enfant avant cinq ans. Paris : Larousse.

Gerken, L. (1987). Telegraphic speaking does not imply telegraphic listening. Stanford Papers and Reports in Child Language Development, 26, 48-55.

Gopnik, M. (1990). Feature blindness : A case study. Language acquisition, 1(2), 139-164.

Hudelot, C. \& Salazar Orvig, A. (2004). Conduites langagières du jeune enfant. In Marcos , H. et al. (éd.), Apprendre à parler : influence du mode de garde, Paris : L'Harmattan, 45-78.

Hurtig, M., Rondal, J.-A. (1999). Introduction à la psychologie de l'enfant. Bruxelles : Mardaga.

Karmiloff-Smith, A.D. (1979). A functional approach to child language: a study of determiners and reference. Cambridge : Cambridge University Press.

Karmiloff, K., Karmiloff-Smith, A. (2001). Comment les enfants entrent dans le langage. Paris : Retz.

Kilani-Schoch, M., de Marco, A., Christofidou, A., Vassilakou, M., Vollman, R. \& Dressler, W.U. (1997). On the demarcation of phases in early morphology acquisition in four languages. Poznan Studies in Contemporary Linguistics, 33 .

Leroy, M., Morgenstern, A., Caët, S. (sous presse). La rectification chez l'enfant : appropriation et internalisation du langage. In La rectification. Ouvrage en hommage à Mary-Annick Morel. Ophrys.

Leroy, M., Martel, K. (sous presse). La communication avant le langage : intonation et mimo-gestualité dans le développement normal et pathologique. In Maillart, C. (éd.), Le développement prélinguistique. Cortil-Wodon : Editions EME - Proximités Logopédie.

McWhinney,B. (2000). The CHILDES Project: Tools for analyzing talk (3rd ed.,Vol.2). Mahwah,NJ : Lawrence Erlbaum Associates.

Marcus, G.F., Pinker, S., Ullman, M., Hollander, M., Rosen, T.J., Xu, F. (1992). Overregularization in language acquisition. Monographs of the Society for Research in Child Development, 57, 34-69.

Morgenstern, A. (2009). L'enfant dans la langue. Paris : Presses de la Sorbonne Nouvelle.

Morgenstern, A., Sekali, M. (1997). L'acquisition des premières prépositions chez un enfant francophone. Faits de Langues, 9, Paris : Ophrys, 201-211.

Nelson, K. (1981). Individual differences in Language Development : Implications for Development and Language. Developmental Psychology, vol.17, $n^{\circ} 2,170-187$.

Peters, A. (1977). Language learning strategies : Does the whole equal the sum of the parts ?. Language, 53, 560-573.

Peters, A. (2001). Fillers syllables : what is their status in emerging grammar ?. Journal of Child Language, vol.28, 229-242.

Rondal, J.-A., Seron, X. (2000). Troubles du langage: bases théoriques, diagnostic et rééducation. Bruxelles : Mardaga.

Roulet, L., Gonin, C., Rigaut, C., Jakubowicz, C. (2004). Le calcul syntaxique de l'accord de genre entre le déterminant et le nom : les enfants dysphasiques diffèrent-ils des enfants normaux ?. In Ferrand \& Grainger (éd.), Psycholinguistique cognitive, Bruxelles : De Boeck \& Larcier, 331-347.

Slobin, D.I. (2001). Form-function relations : how do children find out what they are?. In Bowerman, M. \& Levinson, S.C. (eds), Language acquisition and conceptual development, Cambridge University Press (réédition 2003).

Sourdot, M. (1977). Identification et différenciation des unités. In François, F. (éd.), La syntaxe de l'enfant avant cinq ans, Paris : Larousse, 89-118.

Tomasello, M. (2003). Constructing a language. Harvard University Press. 
978-2-7598-0534-1, Paris, 2010, Institut de Linguistique Française

Psycholinguistique et acquisition

DOI $10.1051 / \mathrm{cmlf} / 2010111$

Veneziano E., Sinclair, H. (2000). The Changing Status of «Filler Syllables» on the way to Grammatical Morphemes. Journal of Child Language, 17, 461-500. 